\title{
ON CONTRACTIONS WITHOUT DISJOINT INVARIANT SUBSPACES
}

\author{
KATSUTOSHI TAKAHASHI \\ (Communicated by Paul S. Muhly) \\ Dedicated to Professor Shozo Koshi on his sixtieth birthday
}

\begin{abstract}
Assume $T$ is a contraction with the following property: there exists an operator $X$ with dense range such that $X T=W X$ where $W$ is a bilateral shift. We give a necessary and sufficient condition that $T$ has no disjoint invariant subspaces.
\end{abstract}

In [3] and [4], Olin and Thomson investigated subnormal operators without disjoint invariant subspaces. They called a (bounded linear) operator $T$ on a Hilbert space cellular-indecomposable if the intersection of any two nonzero invariant subspaces of $T$ is nonzero. In this note we extend the result for cellular-indecomposable subnormal contractions proved in [4].

Let $T$ be a contraction on a separable Hilbert space $\mathscr{H}$, and suppose that $T$ is not of class $C_{0}$; that is, $\lim _{n \rightarrow \infty}\left\|T^{n} x\right\| \neq 0$ for some $x \in \mathscr{H}$. It follows that there exist an isometry $V$ and an operator $X$ with dense range such that $X T=V X$ (see [5, Proposition II.3.5]). Kerchy [2] proved that if this $V$ is a bilateral shift, then $T$ has a nontrivial invariant subspace. (Note that if $V$ is not unitary, then it can be replaced by a bilateral shift.)

Theorem. Let $T$ be a contraction on $\mathscr{H}$ and assume that there exists an operator $X$ with dense range such that $X T=W X$ where $W$ is the bilateral shift on $L^{2}$. Then $T$ is cellular-indecomposable if and only if there exists a quasiaffinity (i.e. an injection with dense range) $Y$ such that $Y T=S Y$ where $S$ is the unilateral shift on $H^{2}$.

The existence of the operator $X$ in the theorem is equivalent to the condition that $\Theta_{T}(\zeta)^{*}$ is not isometric a.e. on the unit circle $\mathbb{T}$ where $\boldsymbol{\Theta}_{T}$ is the characteristic function of $T$ (see [7]). If $T$ is a contraction such that $Y T=S Y$ where $Y$ is a quasiaffinity and $S$ is the unilateral shift, then the restriction of $T$ to any of its cyclic invariant subspaces is quasisimilar to $S$ (see [1, Corollary 15]). Therefore our theorem extends the result of [4]. Such contractions were also considered in [8].

Received by the editors March 7, 1989.

1980 Mathematics Subject Classification (1985 Revision). Primary 47A45.

(C) 1990 American Mathematical Society $0002-9939 / 90 \$ 1.00+\$ .25$ per page 
Proof of theorem. Assume that there exists a quasiaffinity $Y$ such that $Y T=$ $S Y$. Let $\mathscr{M}_{1}$ and $\mathscr{M}_{2}$ be two nonzero invariant subspaces of $T$. For $i=1,2$, there exists an injection $K_{i}$ such that $K_{i} S=T K_{i}$ and $\operatorname{ran} K_{i} \subseteq \mathscr{M}_{i}$ (see [1, Lemma 3]). Since $Y K_{i}$ commutes with $S$, we have $Y K_{i}=f_{i}(S)$ for a nonzero function $f_{i} \in H^{\infty}$. (For a contraction $A$ whose unitary part is absolutely continuous or acts on the space $\{0\}$ and $f \in H^{\infty}, f(A)$ is an operator obtained by the Sz.-Nagy and Foias functional calculus [5].) It follows from the injectivity of $Y$ that $Y\left(\mathscr{M}_{1} \cap \mathscr{M}_{2}\right)=Y \mathscr{M}_{1} \cap Y \mathscr{M}_{2}$. Therefore we have

$$
\begin{aligned}
Y\left(\mathscr{M}_{1} \cap \mathscr{M}_{2}\right) & \supseteq Y K_{1} H^{2} \cap Y K_{2} H^{2}=f_{1} H^{2} \cap f_{2} H^{2} \\
& \supseteq f_{1} f_{2} H^{2} \neq\{0\},
\end{aligned}
$$

and $\mathscr{M}_{1} \cap \mathscr{M}_{2} \neq\{0\}$.

Conversely, we assume that $T$ is cellular-indecomposable. Obviously $T$ is completely nonunitary. By the proof of the theorem in [7] (see [2] for a special case), it follows from the existence of the operator $X$ that there are an operator $Z$ and a vector $x_{0}$ satisfying the following conditions:

(1) $Z T=W Z$ and

(2) the function $\log \left|Z x_{0}\right|$ is integrable.

The condition (2) implies $Z x_{0}=u g$ where $|u|=1$ a.e. and $g$ is an outer function in $H^{2}$, and so $W \mid\left(Z \mathscr{M}_{0}\right)^{-}$is a unilateral shift of multiplicity one where $\mathscr{M}_{0}=\bigvee_{n \geq 0} T^{n} x_{0}$. Since $Z \mid \mathscr{M}_{0}$ is injective (see the proof of [1, Corollary $15]), \operatorname{ker} Z \cap \mathscr{M}_{0}=\{0\}$. Since the subspaces $\operatorname{ker} Z$ and $\mathscr{M}_{0}$ are invariant under $T$ and $T$ is cellular-indecomposable, it follows that $\operatorname{ker} Z=\{0\}$.

We claim that for every $x \in \mathscr{H}$, there exists a nonzero $f \in H^{\infty}$ such that $Z f(T) x \in\left(Z \mathscr{M}_{0}\right)^{-}$. For this purpose, take $x \in \mathscr{H}$ and let $\alpha=\{\zeta \in$ $\left.\mathbb{T}:|(Z x)(\zeta)| \geq\left|\left(Z x_{0}\right)(\zeta)\right|\right\}$. Let us choose a function $h \in H^{\infty}$ such that $|h(\zeta)|=$ $1 / 2$ for $\zeta \in \alpha$ and $|h(\zeta)|=2$ for $\zeta \in \mathbb{T} \backslash \alpha$. Then on $\alpha$ we have

$$
\begin{aligned}
\left|Z x+h Z x_{0}\right| & \geq|Z x|-|h|\left|Z x_{0}\right| \\
& \geq(1-|h|)\left|Z x_{0}\right|=(1 / 2)\left|Z x_{0}\right|,
\end{aligned}
$$

and on $\mathbb{T} \backslash \alpha$ we have

$$
\begin{aligned}
\left|Z x+h Z x_{0}\right| & \geq|h|\left|Z x_{0}\right|-|Z x| \\
& \geq(|h|-1)\left|Z x_{0}\right|=\left|Z x_{0}\right| .
\end{aligned}
$$

Thus $\log \left|Z x+h Z x_{0}\right|$ is integrable, and so $Z x+h Z x_{0}=v k$ where $|v|=1$ a.e. and $k$ is outer. Then, setting $\mathscr{M}=\bigvee_{n \geq 0} T^{n}\left(x+h(T) x_{0}\right)$, we have

$$
u H^{2} \cap v H^{2} \supseteq Z \mathscr{M}_{0} \cap Z \mathscr{M} \supseteq Z\left(\mathscr{M}_{0} \cap \mathscr{M}\right) \neq\{0\}
$$

because $T$ is cellular-indecomposable. Thus there exists a nonzero function $f \in H^{\infty}$ such that $\bar{u} v f \in H^{\infty}$. Then we have

$$
\begin{aligned}
Z f(T) x & =f Z x=f\left(v k-h Z x_{0}\right) \\
& =u(\bar{u} v f k-h f g) \in u H^{2}=\left(Z \mathscr{M}_{0}\right)^{-} .
\end{aligned}
$$

This establishes the claim. 
Let $\mathscr{N}=\left\{x \in \mathscr{H}: Z x \in\left(Z \mathscr{M}_{0}\right)^{-}\right\}$, which is an invariant subspace of $T$. Let $T_{1}=P T \mid \mathscr{H} \ominus \mathscr{N}$ where $P$ is the orthogonal projection onto $\mathscr{H} \ominus \mathscr{N}$. The claim given above implies that for every $x \in \mathscr{H} \ominus \mathscr{N}$, there exists a nonzero function $f \in H^{\infty}$ such that $f\left(T_{1}\right) x=0$, and so $T_{1}$ is of class $C_{0}$ by [6]. That is, there exists an inner function $q$ such that $q\left(T_{1}\right)=0$. Then $q(W) Z \mathscr{H} \subseteq\left(Z \mathscr{M}_{0}\right)^{-}$. Therefore $W \mid(q(W) Z \mathscr{H})^{-}$is unitarily equivalent to the unilateral shift $S$. Since $q(W) Z$ is injective, it follows that there is a quasiaffinity $Y$ such that $Y T=S Y$.

\section{REFERENCES}

1. H. Bercovici and K. Takahashi, On the reflexivity of contractions on Hilbert space, J. London Math. Soc. (2) 32 (1985), 149-156.

2. L. Kerchy, Invariant subspaces of $C_{1}$-contractions with nonreductive unitary extensions, Bull. London Math. Soc. 19 (1987), 161-166.

3. R. F. Olin and J. E. Thomson, Cellular-indecomposable subnormal operators, Integral Equations Operator Theory 7 (1984), 392-430.

4. __, Cellular-indecomposable subnormal operators II, Integral Equations Operator Theory 9 (1986), 600-609.

5. B. Sz.-Nagy and C. Foias, Harmonic analysis of operators on Hilbert space, North-Holland, Amsterdam, 1970.

6. _ L L L L Lal characterization of operators of class $C_{0}$, J. Funct. Anal. 8 (1971), 76-81.

7. K. Takahashi, The reflexivity of contractions with nonreductive $*$-residual parts, Michigan Math. J. 34 (1987), 153-159.

8. __, On quasiaffine transforms of unilateral shifts, Proc. Amer. Math. Soc. 100 (1987), 683-687.

Department of Mathematics, Faculty of Science (General Education), Hokkaido UniVERSITY, SAPPORO 060, JAPAN 